\title{
Association of heat shock proteins with Parkinson's disease
}

\author{
Linda Broer $\cdot$ Peter J. Koudstaal $\cdot$ Najaf Amin $\cdot$ Fernando Rivadeneira $\cdot$ \\ Andre G. Uitterlinden - Albert Hofman • Ben A. Oostra - Monique M. B. Breteler • \\ M. Arfan Ikram • Cornelia M. van Duijn
}

Received: 17 October 2011/Accepted: 14 November 2011/Published online: 26 November 2011

(C) The Author(s) 2011. This article is published with open access at Springerlink.com

Parkinson's disease (PD) is the most common neurodegenerative movement disorder, characterized by progressive degeneration of dopaminergic neurons in the substantia nigra and the presence of Lewy bodies in the affected neurons, comprised of aggregated $\alpha$-synuclein $[1,2]$. PD can therefore be considered an example of a protein-folding disorder [3]. Heat Shock Proteins (HSPs) are the major chaperones mediating (re) folding of proteins, ensuring that these proteins stay in their native formations during conditions of stress to the cell [4]. We tested 27 genes encoding all members of the HSP10, HSP60, HSP70, HSP90 and the heat shock factors (HSF) families and assessed their association with PD.

Our discovery cohort was the Rotterdam Study (RS), which is a population-based cohort study that investigates the occurrence and determinants of diseases in the elderly [5]. The Medical Ethics Committee at Erasmus Medical Center approved the study protocol. Genomic DNA was

Electronic supplementary material The online version of this article (doi:10.1007/s10654-011-9635-9) contains supplementary material, which is available to authorized users.

L. Broer · N. Amin · F. Rivadeneira - A. G. Uitterlinden .

A. Hofman - M. M. B. Breteler · M. A. Ikram .

C. M. van Duijn ( $\square)$

Department of Epidemiology, Erasmus University Medical

Center, Dr. Molewaterplein 50, PO-Box 2040,

3000 CA Rotterdam, The Netherlands

e-mail: c.vanduijn@erasmusmc.nl

P. J. Koudstaal

Deparment of Neurology, Erasmus Medical Center, Rotterdam,

The Netherlands

F. Rivadeneira - A. G. Uitterlinden · B. A. Oostra Department of Internal Medicine, Erasmus Medical Center, Rotterdam, The Netherlands extracted from whole blood samples using standard methods [6]. Genome-wide SNP genotyping was performed using Infinium II assay on the HumanHap550 Genotyping BeadChips (Illumina Inc, San Diego, USA). Approximately 2 million SNPs were imputed using release 22 HapMap CEU population as reference. The imputations were performed using MACH software (http://www.sph. umich.edu/csg/abecasis/MACH/).

A total 3,828 SNPs in 27 genes were initially selected for the association test on the basis of the following criteria: (1) position within the genes of interest with a margin of $100 \mathrm{~kb}$ on each side of the genes according to NCBI build 36.3, (2) p-value for Hardy-Weinberg equilibrium test $\geq 0.0001$, and (3) call rate $\geq 95 \%$. For further analysis and selection of SNPs for replication analysis only SNPs with an imputation quality greater than $80 \%$ and a minor allele frequency (MAF) higher than 0.05 were selected.

We analyzed the individual SNPs using ProbABEL [7]. We used allele-based logistic regression to test the association between a single SNP and PD. Odds Ratios (ORs) for each SNP were derived adjusting for age and sex. To calculate empirical significance for SNPs, permutations were performed per region of interest (ROI). Briefly, we performed 10,000 replications with randomly allocated phenotypes. To estimate empirical significance each observed test statistic was compared with null statistics obtained empirically and the $P$-value was estimated as the proportion of replicas generating the test statistics greater than or equal to the observed statistic.

For each suggestively associated ROI $\left(P_{\text {permuted }}<0.10\right)$ we next selected the 'independently associated' SNPs in a backward stepwise logistic regression until only nominally significant SNPs remained in the model. These independently associated SNPs were then followed up. Further suggestively associated SNPs inside the gene were also sent 
for replication. For replication we used online available genome wide association studies (GWAS) on PD and selected the study from Simon-Sanchez et al. [8] published in Nature Genetics in 2009. This study included a total of 5,197 PD cases and 8,803 controls. In their supplementary tables, the authors present $P$-values and effect estimates for all SNPs with a $P$-value lower than $1 \times 10^{-4}$ in the discovery stage.

General characteristics of the discovery population are shown in Table 1. A total of 134 cases of Parkinson Disease were identified. The cases are significantly older than controls. A summary of all SNPs tested in the HSP genes in the discovery phase can be found in Supplementary Table 1. As HSP10 (HSPEl) and HSP60 (HSPDI) are located head to head on chromosome 2 these were taken as 1 Region of Interest (ROI) with 52 SNPs (Chaperonin). The same applies to 3 HSP70 genes located on chromosome 6 with 148 SNPs (HSPA1).

Figure 1 shows an overview of the $P$-values in all genes tested in RS1. After adjusting for multiple testing by permutation analysis, 3 genes still showed evidence for assocation to PD in RS1. These genes encode Chaperonin, HSP70 member HSPA13 and HSF member HSF5. For replication the following SNPs were selected: rs17730989 and rs788016 for Chaperonin, rs2822686 for HSPA13 and

Table 1 General characteristics for cases and controls in the discovery cohort

\begin{tabular}{lll}
\hline & Cases & Controls \\
\hline$N$ & 134 & 5,422 \\
Age $($ mean \pm SD $)$ & $74.2 \pm 8.0$ & $68.5 \pm 8.5$ \\
Gender $(\%$ female $)$ & 58.5 & 53.0 \\
\hline
\end{tabular}

rs9889631 for HSF5. Two SNPs in Chaperonin were selected as both were intronic SNPs for HSPD1. The results for the SNPs we attempted to replicate are in Table 2. None of these SNPs had a $P$-value lower than $1 \times 10^{-4}$ in our replication sample. We did not find significant evidence for an association between SNPs in or directly flanking the HSP genes with PD.

Chaperonin was the best candidate for association with PD to reach significance in the discovery cohort, but none of the SNPs in HSPDI were replicated. Chaperonin is an interesting candidate for association with $\mathrm{PD}$ as it is the major folding machine of the mitochondria [9]. Its important influence on mitochondrial function is underlined by findings that yeast cells carying a null mutation in HSP60 have severe defects in folding of mitochondrial proteins and are non-viable [10]. In contrast, yeast cells with conditional mutations in HSP60 tend to accumulate misfolded proteins that are unable to perform their functions, similar to $\alpha$-synculein aggregation in PD [10].

The importance of mitochondrial dysfunction in PD is widely accepted. All major genes previously identified for familial PD influence mitochondrial functioning [11]. The importance of HSPDI in mitochondrial functioning could be an explanation for the absence of an association with PD. Only variants with very small ORs are to be expected in such a critical gene, leaving our study underpowered to reach statistical significance.

In summary, we examined associations between 3,828 SNPs in and around 27 genes encoding the Chaperonin, HSP70, HSP90 and HSF gene families in two unrelated cohorts, and found no statistically significant associations with PD. Despite evidence on the protein level for involvement of these genes, no large-scale studies have
Fig. 1 Summary results of all SNPs tested. The $-\log (P$-values $)$ of all SNPs tested are presented, where the SNPs are sorted on the genes on $\mathrm{x}$-axis. The horizontal lines show the $-\log (P$-values $)$ of $0.05,0.01$ and 0.001 respectively

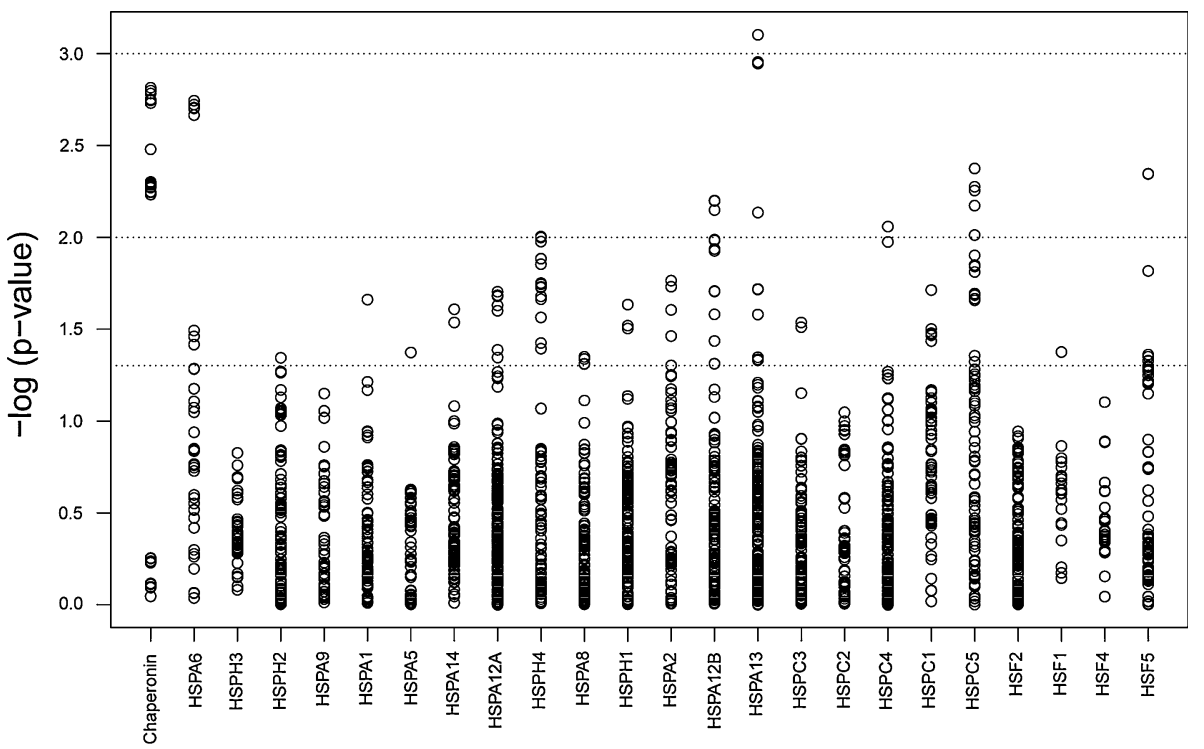

Genes 
Table 2 Association of the SNPs significantly associated with PD in the Rotterdam Study

\begin{tabular}{|c|c|c|c|c|c|c|c|c|c|c|}
\hline \multirow[t]{2}{*}{ Gene } & \multirow[t]{2}{*}{ SNP } & \multirow[t]{2}{*}{ Chr. } & \multirow[t]{2}{*}{ Position } & \multirow[t]{2}{*}{ RA } & \multicolumn{3}{|c|}{ Rotterdam Study } & \multicolumn{3}{|c|}{ Simon-Sanchez et al. [8] } \\
\hline & & & & & Freq. & OR & $95 \% \mathrm{CI}$ & Freq. & OR & $95 \% \mathrm{CI}$ \\
\hline HSPD1 & rs17730989 & 2 & $198,070,769$ & $\mathrm{C}$ & 0.49 & 0.67 & $0.52-0.86$ & NF & NF & NF \\
\hline HSPD1 & rs788016 & 2 & $198,060,538$ & G & 0.49 & 0.68 & $0.53-0.87$ & NF & NF & NF \\
\hline HSPA13 & rs 2822686 & 21 & $14,773,971$ & $\mathrm{~T}$ & 0.82 & 0.61 & $0.46-0.8$ & NF & NF & NF \\
\hline HSF5 & rs9889631 & 17 & $53,985,358$ & $\mathrm{~T}$ & 0.93 & 0.55 & $0.37-0.81$ & NF & NF & NF \\
\hline
\end{tabular}

$R S 1$ Rotterdam study, $C h r$ chromosome, $R A$ risk allele, Freq frequency, $O R$ odds ratio, $95 \%$ CI 95\% confidence interval, $N F$ not found with a $P$-value of $10^{-4}$

been performed on the role of HSPs in patients with PD, until now.

Acknowledgments The generation and management of GWAS genotype data for the Rotterdam Study is supported by the Netherlands Organisation of Scientific Research NWO Investments (nr. 175.010.2005.011, 911-03-012). This study is funded by the Research Institute for Diseases in the Elderly (014-93-015; RIDE2), the Netherlands Genomics Initiative (NGI)/Netherlands Organisation for Scientific Research (NWO) project nr. 050-060-810. We thank Pascal Arp, Mila Jhamai, Marijn Verkerk, Lizbeth Herrera and Marjolein Peters for their help in creating the GWAS database, and Karol Estrada and Maksim V. Struchalin for their support in creation and analysis of imputed data. The Rotterdam Study is funded by Erasmus Medical Center and Erasmus University, Rotterdam, Netherlands Organization for the Health Research and Development (ZonMw), the Research Institute for Diseases in the Elderly (RIDE), the Ministry of Education, Culture and Science, the Ministry for Health, Welfare and Sports, the European Commission (DG XII), and the Municipality of Rotterdam. The authors are grateful to the study participants, the staff from the Rotterdam Study and the participating general practitioners and pharmacists. This study was further supported by the International Parkinson Fonds.

Open Access This article is distributed under the terms of the Creative Commons Attribution Noncommercial License which permits any noncommercial use, distribution, and reproduction in any medium, provided the original author(s) and source are credited.
2. Dawson TM, Dawson VL. Molecular pathways of neurodegeneration in Parkinson's disease. Science. 2003;302(5646):819-22. doi:10.1126/science.1087753302/5646/819.

3. Selkoe DJ. Folding proteins in fatal ways. Nature. 2003;426(6968): 900-4. doi:10.1038/nature02264nature02264.

4. Chen S, Brown IR. Neuronal expression of constitutive heat shock proteins: implications for neurodegenerative diseases. Cell Stress Chaperones. 2007;12(1):51-8.

5. Hofman A, van Duijn CM, Franco OH, Ikram MA, Janssen HL, Klaver CC, et al. The Rotterdam Study: 2012 objectives and design update. Eur J Epidemiol. 2011;26(8):657-86. doi:10.1007/ s10654-011-9610-5.

6. Miller SA, Dykes DD, Polesky HF. A simple salting out procedure for extracting DNA from human nucleated cells. Nucleic Acids Res. 1988;16(3):1215.

7. Aulchenko YS, Struchalin MV, van Duijn CM. ProbABEL package for genome-wide association analysis of imputed data. BMC Bioinform. 2010;11:134. doi:10.1186/1471-2105-11-134.

8. Simon-Sanchez J, Schulte C, Bras JM, Sharma M, Gibbs JR, Berg $\mathrm{D}$, et al. Genome-wide association study reveals genetic risk underlying Parkinson's disease. Nat Genet. 2009;41(12):1308-12. doi:10.1038/ng.487.

9. Ostermann J, Horwich AL, Neupert W, Hartl FU. Protein folding in mitochondria requires complex formation with hsp60 and ATP hydrolysis. Nature. 1989;341(6238):125-30. doi:10.1038/341125a0.

10. Cheng MY, Hartl FU, Martin J, Pollock RA, Kalousek F, Neupert $\mathrm{W}$, et al. Mitochondrial heat-shock protein hsp60 is essential for assembly of proteins imported into yeast mitochondria. Nature. 1989;337(6208):620-5. doi:10.1038/337620a0.

11. Abou-Sleiman PM, Muqit MM, Wood NW. Expanding insights of mitochondrial dysfunction in Parkinson's disease. Nat Rev Neurosci. 2006;7(3):207-19. doi:10.1038/nrn1868.

\section{References}

1. Baba M, Nakajo S, Tu PH, Tomita T, Nakaya K, Lee VM, et al. Aggregation of alpha-synuclein in Lewy bodies of sporadic Parkinson's disease and dementia with Lewy bodies. Am J Pathol. 1998;152(4):879-84. 